\title{
Experiencia en la aplicación de la docencia inversa para el aprendizaje de la asignatura de Máquinas Hidráulicas (Grado de Ingeniería en Tecnologías Industriales)
}

\section{Rosa Llácer Iglesias y F. Javier Martínez-Solano}

Departamento de Ingeniería Hidráulica y Medio Ambiente, Universitat Politècnica de València. rollaig@hma.upv.es; jmsolano@upv.es.

\begin{abstract}
In this paper, the results of an experience based on the principles of flipped classroom methodology are presented. In particular, the experience has been applied to the labs of the course of Hydraulic Machines given in the 4th year of the Degree in Industrial Technologies Engineering. The flipped classroom was made compulsory for all students enrolled in the course. The scope of the flipped classroom involves both specific topics of the subject and other soft skills such as training in safety and risk prevention. As lessons learned from experienceit is possible to highlight an improvement in academic performance compared to the previous year, although there have also noted areas for improvement.
\end{abstract}

Keywords: laboratory practice, flipped classroom, autonomous learning, engineering

\begin{abstract}
Resumen
En este trabajo se presentan los resultados de la puesta en práctica de metodología basada en los principios de la docencia inversa. En concreto, la experiencia se ha aplicado a las prácticas de laboratorio de la asignatura de Máquinas Hidráulicas impartida en el $4^{\circ}$ curso del Grado en Ingeniería de Tecnologías Industriales, haciéndola extensible a todos los alumnos matriculados de la asignatura. El alcance de la docencia inversa planteada abarca tanto la temática específica de la asignatura como otras temáticas transversales como es el caso de la formación en seguridad y prevención de riesgos laborales. Como conclusiones extraídas de la experiencia destaca una mejora en los resultados académicos con respecto a los del curso anterior, si bien también se han observado áreas de mejora.
\end{abstract}

Palabras clave: prácticas, laboratorio, docencia inversa, aprendizaje autónomo, ingeniería 


\section{Introducción}

Una de las críticas más habituales de los métodos docentes tradicionales es la poca capacidad de estos para implicar al alumno. Así, a partir de unos contenidos previamente establecidos los alumnos deben estudiarlos y aprehenderlos. Estos conocimientos son posteriormente aplicados a casos concretos en los cuales el alumno puede constatar la utilidad de los mismos. Por último el alumno debe estudiar tanto los conocimientos más teóricos como sus aplicaciones teniendo como principal finalidad la superación de una evaluación. En resumen, se trata de una estructura rígida en la que el docente establece los contenidos y sus posibles aplicaciones.

Con la aparición de las nuevas tecnologías se abre un nuevo campo para el autoaprendizaje. Así, en el año 2007 los profesores Sams y Bergmann del Instituto Woodland Park en Colorado (EE.UU.) propusieron un sistema para permitir que los alumnos que perdían clases pudieran recuperarlas en los momentos que ellos decidieran a partir de grabaciones. A partir de esta experiencia y los resultados obtenidos se acuñó el término flip classroom (Sams y Bergmann, 2013), traducido al español como docencia inversa.

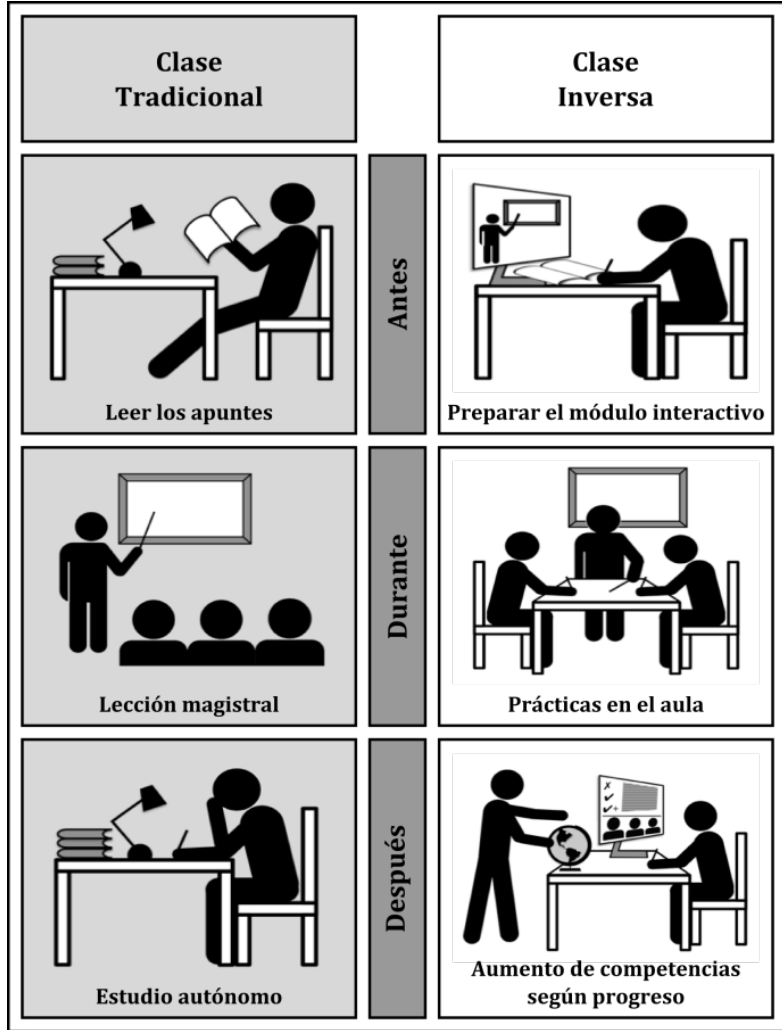

Fig. 1 Comparación entre la docencia tradicional y la docencia inversa (adaptado de Rhode, 2016).

Las diferencias entre una clase tradicional y una clase inversa se resumen en la Figura 1 (Rhode, 2015). A diferencia de la estructura de una clase tradicional antes comentada, en la clase inversa el material se prepara para ser estudiado de manera autónoma. Si bien se da por hecho que se ha de tratar de material audivisual, hay que recordar que su origen está en la 
sucesión de diapositivas y explicaciones en la pizarra. Una vez completado este trabajo previo, el alumno tiene unos conocimientos básicos que serán aplicados en el aula con ayuda del profesor. De esta forma, la clase pasa a estar basada en la resolución de casos prácticos. Finalmente, en la medida que los alumnos van ampliando conocimientos, las aplicaciones van siendo cada vez más complejas. A mayor dedicación del alumno, más competencias va adquiriendo. Esto elimina una de las principales limitaciones del sistema tradicional: la falta de diferenciación del alumnado.

Durante el ejercicio profesional de la ingeniería industrial, además de los conocimientos técnicos tradicionales se valora cada vez más otras capacidades o competencias, como la capacidad de autoaprendizaje o el trabajo colaborativo. Esta capacidad presumiblemente les facilitará la adaptación al entorno laboral específico en que se puedan encontrar, entorno por otra parte siempre cambiante, ayudándoles asimismo a iniciar el camino a un posterior crecimiento profesional.

En este contexto la metodología de docencia inversa ofrece una alternativa especialmente interesante a la docencia tradicional, ya que, de una forma u otra, puede contribuir en mayor grado a la adquisición de este tipo de competencias. Del mismo modo, puede ayudar a mejorar la eficiencia en la gestión del tiempo, cualidad igualmente deseable en un ingeniero industrial. Si bien es cierto que, este cambio de metodología, requiere un esfuerzo importante, tanto por parte del alumno como del equipo docente.

Por otra parte, resulta evidente que las nuevas tecnologías permiten ofrecer una serie de herramientas y opciones que indudablemente son de gran utilidad en la docencia inversa. No obstante, probablemente un cierto porcentaje del personal docente puede llegar a percibir esto incluso como una barrera e incluso muchos de ellos considerar el esfuerzo requerido en el salto de un modelo a otro como excesivo y al mismo tiempo arriesgado en cuanto a resultados. Sin embargo, no tiene porqué ser imprescindible recurrir a toda clase de herramientas audiovisuales ni desarrollar estructuras, plataformas y materiales de apoyo complejos y/o sofisticados para empezar a aplicar esta metodología docente.

La técnica de la clase o docencia inversa surgió para dar respuesta a las distintas necesidades de los alumnos de educación primaria y secundaria (Sams y Bergmann, 2013). Sin embargo, en los últimos años está creciendo notablemente en el ámbito universitario el interés en la aplicación de metodologías activas de aprendizaje, conscientes de que la formación que deben recibir los alumnos a lo largo de sus estudios debe ir más allá de la adquisición de los conocimientos técnicos necesarios, y contribuir también al desarrollo de habilidades y competencias que les permitan posteriormente desarrollar con éxito su carrera profesional. Así, Argente et al. (2015) describen una prueba piloto realizada en la Universidad Politécnica de Valencia (UPV) en el curso 2014-2015 con alumnos del segundo curso del Grado en Ingeniería Informática. Si bien los resultados del experimento resultaron alentadores, el carácter voluntario del mismo (con apenas un 4\% de los alumnos matriculados tomando parte del mismo) resta representatividad al mismo. Otros autores (Fung, 2015) utilizaron una técnica similar basada en el uso de cámaras subjetivas (tipo Go-Pro®) para la preparación de las prácticas de laboratorio de química. En este caso, el beneficio obtenido fue superior dado el limitado tiempo disponible en general en los laboratorios. Este trabajo intenta, por una

(cc) EY-Nc-ND 2016, Universitat Politècnica de València

Congreso In-Red (2016) 
parte, extender las experiencias realizadas previamente en la UPV a la totalidad de los alumnos y por otra optimizar el tiempo de permanencia en el Laboratorio de Mecánica de Fluidos.

En el presente artículo se describe una experiencia sencilla en un acercamiento a la implantación de la docencia inversa. En concreto, la experiencia se ha aplicado a las prácticas de laboratorio de la asignatura de Máquinas Hidráulicas impartida en el $4^{\circ}$ curso del Grado en Ingeniería de Tecnologías Industriales, haciéndola extensible a todos los alumnos matriculados de la asignatura: en total 330 alumnos divididos en 15 grupos de prácticas. En dicha experiencia se ha pretendido cubrir no solo temática afín a la propia asignatura sino también temáticas transversales, como las relacionadas con la formación en seguridad en el medio laboral (en este caso, en un laboratorio).

\section{Objetivos}

La asignatura objeto de la experiencia es una asignatura obligatoria del Grado en Ingeniería de Tecnologías Industriales (Máquinas Hidráulicas), correspondiente al primer semestre del último curso $\left(4^{\circ} \mathrm{A}\right)$. La asignatura tiene un elevado número de alumnos matriculados (330 en el curso 2015-2016) repartidos en cinco grupos: tres de mañana impartidos en castellano, un grupo de mañana en valenciano y un último grupo con horario vespertino. Cada uno de estos cinco grupos se subdivide en tres para las prácticas dando como resultado 15 grupos de prácticas con un número media de alumnos de 22 alumnos por grupo. La asignatura consta de 4,5 créditos repartidos en 3,6 créditos de clase ordinaria (lección magistral y prácticas en el aula) y 0,9 créditos de prácticas repartidos en tres sesiones de tres horas cada una.

Inicialmente se pensó en llevar a cabo en primera instancia la implantación de la metodología de docencia inversa a la totalidad de la asignatura. Sin embargo, hay tres motivos que sugerían que éste sería un objetivo probablemente demasiado ambicioso. Por un lado, el contenido técnico propio de la asignatura hacía peligrar los resultados globales de la asignatura ante un cambio tan sustancial en la metodología. En este sentido, los profesores de la asignatura tienen publicado un libro en el Servicio de Publicaciones de la UPV (López Patiño y Martínez Solano, 2001) que recoge la totalidad del temario. Por otro lado, el elevado número de alumnos matriculados suponía una labor de coordinación y seguimiento consecuente con el mismo. Por último, la necesidad de inversión en recursos tanto en tiempo como en personal. Sin embargo, el interés del equipo docente por avanzar en esa dirección hizo que se plantease llevarlo a cabo, a modo de experiencia piloto, en una pequeña pero significativa parte de la asignatura, por lo que se eligió para ello la parte de prácticas de laboratorio. Esto permitiría sacar mayor provecho al tiempo de permanencia en el laboratorio, utilizando los conceptos de la docencia inversa a la preparación de las prácticas de forma que los alumnos llegaran al laboratorio con los conocimientos relativos a normas de seguridad y procedimientos a seguir ya revisados.

Por tanto, el principal objetivo de esta propuesta es el de dar un primer paso hacia la implantación de la metodología de docencia inversa en la asignatura de Máquinas Hidráulicas, de momento limitada a las prácticas de laboratorio.

(cc) BY-NC-ND 2016, Universitat Politècnica de València 
Para alcanzar este objetivo general se definieron una serie de objetivos específicos:

- Definir el material necesario para esta fase.

- Diseñar el material requerido.

- $\quad$ Establecer los hitos o puntos de control necesarios.

- Realizar un análisis de los resultados para introducción de mejoras en el próximo curso y ampliación a otras partes de la asignatura.

- Compartir la experiencia para animar a otros docentes a iniciar desarrollos en la misma línea en sus áreas de conocimiento.

\section{Desarrollo de la innovación}

Las prácticas de laboratorio de Máquinas Hidráulicas consisten básicamente en la realización de ensayos en diferentes montajes de circuitos hidráulicos sencillos para caracterización de bombas y aplicación de algunos conceptos básicos de su funcionamiento y operación. Dichos ensayos se realizan en equipo, formando como máximo 3 grupos de entre 4 y 10 alumnos, variable en función del número total de asistentes a la sesión (teniendo en cuenta que cada grupo de teoría se desdobla en 3 grupos de prácticas, en una sesión está prevista como máximo la asistencia de 30 alumnos).

Hasta el curso anterior al de la experiencia, para cada grupo dichas prácticas constaban de 2 sesiones de 2,5 horas de duración cada una, en las que se llevaban a cabo 2 ó 3 ensayos en total. En función del número y predisposición de los alumnos asistentes a cada sesión, el ritmo de las mismas podía ser muy diferente, produciéndose además en muchos casos "tiempos muertos", que por lo general, no eran aprovechados por los alumnos. Por otra parte, como suele ser habitual en muchas prácticas en las carreras de ingeniería, a pesar de que los guiones de prácticas se encuentren disponibles con anterioridad a las mismas, la experiencia muestra que la gran mayoría de alumnos no suele prepararlas previamente. De este modo, se consume al inicio de las sesiones una importante cantidad de tiempo en la exposición de contenidos y metodología, produciéndose también durante el desarrollo de los ensayos tiempos inactivos por no tener clara la operativa a seguir por parte del alumno, e incluso el objetivo a conseguir.

En este contexto, las prácticas de laboratorio parecían mostrarse como la parte de la asignatura que podía encontrar mayores beneficios en la aplicación piloto de la metodología de docencia inversa. Además de fomentar el autoaprendizaje, con su implantación se ha intentado paralelamente que el tiempo que el alumno permanece en el laboratorio sea aprovechado al máximo. De este modo, el desarrollo de los ensayos se ha concentrado en una única sesión de 3 horas y se ha elaborado nuevo material que facilitase la preparación previa, el apoyo durante su realización y análisis de resultados posterior.

\subsection{Definición del material}

(c) EY-NC-ND 2016, Universitat Politècnica de València

Congreso In-Red (2016) 
Al hablar de docencia inversa es habitual pensar en material audiovisual, frecuentemente videos de corta duración con contenidos concretos. Si bien para la exposición de contenidos teóricos es ciertamente un medio muy recomendable a tener en cuenta, en este caso se consideró más adecuado, para el objetivo final establecido, la adaptación de material. Sams y Bergmann (2013), considerados los pioneros en esta metodología, diferencian entre la idea que se persigue y los medios utilizados para alcanzarla. Así, hay alumnos que valoran el material audiovisual preparado mientras que otros prefieren materiales convencionales o, simplemente, una guía de estudios. Puesto que se pretende fomentar el aprendizaje autónomo en el ejercicio profesional de la ingeniería industrial, familiarizarse con la lectura y comprensión de instrucciones de operación y manuales de maquinaria se ha considerado de particular interés. Con esa misma finalidad y por su importancia en el ámbito profesional en el que se moverán, se ha incluido además, por primera vez como parte del contenido, el análisis de riesgos y las normas de seguridad en el laboratorio.

Tal y como se ha indicado anteriormente, los guiones de prácticas se elaboraron de forma que sirviesen de material de apoyo antes, durante y después de la sesión en el laboratorio. De este modo la documentación se estructuró en 3 partes:

- Parte I: Normas de seguridad y prevención de riesgos en el ambiente de trabajo.

- Parte II: Introducción, descripción y procedimiento de ensayos.

- Parte III: Datos experimentales, cálculos y análisis de resultados.

Cada una de estas partes lleva relacionada unos anexos, de una página cada uno, con cuestiones cortas para su evaluación. De este modo, para proceder con los ensayos en el laboratorio era requisito indispensable que los anexos de las partes I y II (relacionados con la seguridad y los procedimientos de operación) se trajesen cumplimentados a mano y de forma individual, y fuesen entregados al inicio de la práctica. Por su parte, los anexos de la parte III (cálculos y análisis de resultados) debían ser cumplimentados durante el desarrollo de la práctica, en grupos, y entregarse al finalizar la sesión. Todo el material se colgó en la plataforma de comunicación entre profesores y alumnos de la UPV (PoliformaT) con antelación suficiente, recordando que antes de asistir a la práctica, ésta debía ser preparada y se debían trabajar los aspectos necesarios para el desarrollo de la misma. Se avisó además que no se permitiría realizar la práctica a los alumnos que no hubiesen leído y comprendido la parte I (seguridad) y cumplimentado y entregado el correspondiente anexo, mientras que la entrega correspondiente a la parte II (procedimiento) con retraso implicaría penalización en la nota correspondiente.

La figura 2 muestra la vista de la plataforma PoliformaT que los alumnos podían observar relacionada con la docencia inversa en las prácticas de laboratorio. En todo momento los alumnos tenían acceso al 100\% de la documentación por lo que la realización de los anexos I y II solo exigía la lectura de la documentación correspondiente. 


\begin{tabular}{|c|c|c|c|}
\hline$\square$ Practicas & Acciones - & $\begin{array}{l}\text { Sitio } \\
\text { completo }\end{array}$ & Gonzalo López Patiño \\
\hline G Práctica de Laboratorio & Acciones - & $\begin{array}{l}\text { Sitio } \\
\text { completo }\end{array}$ & $\begin{array}{l}\text { Rosa Maria Llacer } \\
\text { Iglesias }\end{array}$ \\
\hline $\begin{array}{l}\text { 1. PR LAB MAQ HID_SEGURIDAD } \\
\text { 09_2015.pdf }\end{array}$ & Acciones - & $\begin{array}{l}\text { Sitio } \\
\text { completo }\end{array}$ & $\begin{array}{l}\text { Rosa Maria Llacer } \\
\text { Iglesias }\end{array}$ \\
\hline $\begin{array}{l}\text { 적.pd 1.PR LAB MAQ HID_SEGURIDAD_ANEXO } \\
\text { 09_2015.pdf }\end{array}$ & Acciones - & $\begin{array}{l}\text { Sitio } \\
\text { completo }\end{array}$ & $\begin{array}{l}\text { Rosa Maria Llacer } \\
\text { Iglesias }\end{array}$ \\
\hline $\begin{array}{l}\text { D] 2. PR LAB MAQ HID_INTROD+PROCED } \\
\text { 09_2015.pdf }\end{array}$ & Acciones - & $\begin{array}{l}\text { Sitio } \\
\text { completo }\end{array}$ & $\begin{array}{l}\text { Rosa Maria Llacer } \\
\text { Iglesias }\end{array}$ \\
\hline $\begin{array}{l}\text { 7] 2. PR LAB MAQ } \\
\text { HID_INTROD+PROCED_ANEXO 09_2015.pdf }\end{array}$ & Acciones $\nabla$ & $\begin{array}{l}\text { Sitio } \\
\text { completo }\end{array}$ & $\begin{array}{l}\text { Rosa Maria Llacer } \\
\text { Iglesias }\end{array}$ \\
\hline $\begin{array}{c}\text { 3.1 PR LAB MAQ } \\
\text { HID_DATOS+RESULTADOS_ANEXO1 09_2015.pdf }\end{array}$ & Acciones - & $\begin{array}{l}\text { Sitio } \\
\text { completo }\end{array}$ & $\begin{array}{l}\text { Rosa Maria Llacer } \\
\text { Iglesias }\end{array}$ \\
\hline $\begin{array}{l}\text { I } 3.2 \text { PR LAB MAQ } \\
\text { HID_DATOS+RESULTADOS_ANEXO2 09_2015.pdf }\end{array}$ & Acciones - & $\begin{array}{l}\text { Sitio } \\
\text { completo }\end{array}$ & $\begin{array}{l}\text { Rosa Maria Llacer } \\
\text { Iglesias }\end{array}$ \\
\hline $\begin{array}{l}\text { 7. } 3.3 \text { PR LAB MAQ } \\
\text { HID_DATOS+RESULTADOS_ANEXO3 10_2015.pdf }\end{array}$ & Acciones - & $\begin{array}{l}\text { Sitio } \\
\text { completo }\end{array}$ & $\begin{array}{l}\text { Rosa Maria Llacer } \\
\text { Iglesias }\end{array}$ \\
\hline $\begin{array}{l}\text { 3. 3. PR LAB MAQ HID_DATOS+RESULTADOS } \\
\text { 09_2015.pdf }\end{array}$ & Acciones - & $\begin{array}{l}\text { Sitio } \\
\text { completo }\end{array}$ & $\begin{array}{l}\text { Rosa Maria Llacer } \\
\text { Iglesias }\end{array}$ \\
\hline
\end{tabular}

Fig. 2 Conjunto de documentos preparados para la implantación de la metodología de docencia inversa en las prácticas de laboratorio de la asignatura Máquinas Hidráulicas.

\subsection{Diseño del material}

En el diseño del material se tuvo en cuenta que, por supuesto, este debía estar correctamente estructurado, ser claro y conciso, pero completo a la vez, con toda la información necesaria para el desarrollo de la práctica. Pero además, una cuestión primordial, era que debía describir exactamente los equipos e instalaciones a utilizar y ensayos a realizar. En este sentido cabe mencionar, que es habitual que a lo largo de los años se produzcan variaciones en los montajes de prácticas de laboratorio, que hacen que, con frecuencia, los guiones de las mismas presenten variaciones respecto a lo que se va a desarrollar, lo que en ocasiones pueden llegar a confundir a los alumnos. En este caso era fundamental que el material fuese totalmente adaptado a lo que los alumnos iban a encontrarse en el laboratorio y a lo que se debía llevar a cabo.

En este curso los ensayos se realizaron en tres bancos de trabajo, todos ellos circuitos hidráulicos sencillos con bombas. Los alumnos se dividían en tres grupos e iban realizando de forma rotativa los ensayos, de modo que cada grupo ensayaba en un banco de trabajo y al finalizar la sesión todos ellos hubiesen llevado a cabo los tres ensayos. En todos los casos, el objetivo principal es familiarizar al alumno con los métodos de ensayo de una bomba sumergida para la obtención de sus curvas características H-Q, P-Q y ๆ-Q, así como con la instrumentación a emplear y en cada uno de ellos se añade un segundo objetivo específico de ese montaje. En concreto los tres bancos de trabajo consisten en:

- Banco 1: Ensayo de bomba de achique sumergida. Caracterización de la bomba y comparación de la curva obtenida por ajuste con la proporcionada por el fabricante.

- Banco 2: Ensayo de bomba sumergida con variador de frecuencia. Caracterización de la bomba a diferentes frecuencias y aplicación de la teoría de semejanza.

(cc) EY-Nc-ND 2016, Universitat Politècnica de València

Congreso In-Red (2016) 
- Banco 3: Ensayo de dos bombas centrífugas en asociación. Caracterización de una bomba individual y caracterización de la asociación de ambas, en serie y en paralelo.

A continuación se describe más detalladamente la estructura y contenidos desarrollados en el material elaborado.

En la Parte I de prevención y seguridad los contenidos incluían una introducción, una descripción de los riesgos en el laboratorio (éléctricos, mecánicos y químicos) y las normas de seguridad de obligado cumplimiento durante las prácticas. En este caso, las cuestiones formuladas en el anexo correspondiente, que debía entregarse de forma individual y cumplimentado a mano al inicio de la clase, estaban orientadas a asegurar la lectura y comprensión del documento. De este modo, estas cuestiones eran del tipo:

- Riesgos en el laboratorio. ¿ Has entendido los riesgos que existen en el laboratorio? En caso afirmativo resume en 3-4 líneas cuáles son. En caso negativo es importante que consultes tus dudas con el profesor al inicio de la clase de prácticas.

- Normas de seguridad: ¿Has entendido y aceptas las normas establecidas? En caso negativo es importante que consultes tus dudas con el profesor al inicio de la clase de prácticas. ¿Crees que son necesarias? Justifica en 2-3 líneas tu respuesta.

En la Parte II los contenidos incluyen en primer lugar una introducción, en la que se describe la metodología y los fundamentos teóricos generales, comunes a todos los bancos de ensayo. Posteriormente, para cada banco de trabajo, se detalla de forma específica: objetivos, fundamentos teóricos específicos, descripción del banco de trabajo y procedimiento de ensayo. En estos dos últimos apartados se incluyó información completa, a modo de manual de instrucciones y para ello fue fundamental incluir fotografías actuales de los equipos con señalización de los componentes, tal y como se muestra en las figuras 3 y 4 mostradas en la página siguiente. Al igual que en la parte I, las cuestiones formuladas en el anexo correspondiente, también debían entregarse de forma individual y cumplimentadas a mano al inicio de la sesión, y estaban orientadas a asegurar la lectura y comprensión del documento.

Por último, en la Parte III los contenidos incluyen para cada banco de trabajo, una tabla de recogida de datos experimentales, una tabla de cálculos, análisis de resultados y cuestiones complementarias, haciendo hincapié en todos los casos en la obtención de conclusiones, análisis crítico de resultados e identificación de posibles fuentes de error. En esta parte hay tres anexos, uno para cada banco de trabajo, con resumen de resultados y conclusiones en cada caso, que debían ser entregados por grupos al finalizar la sesión. 


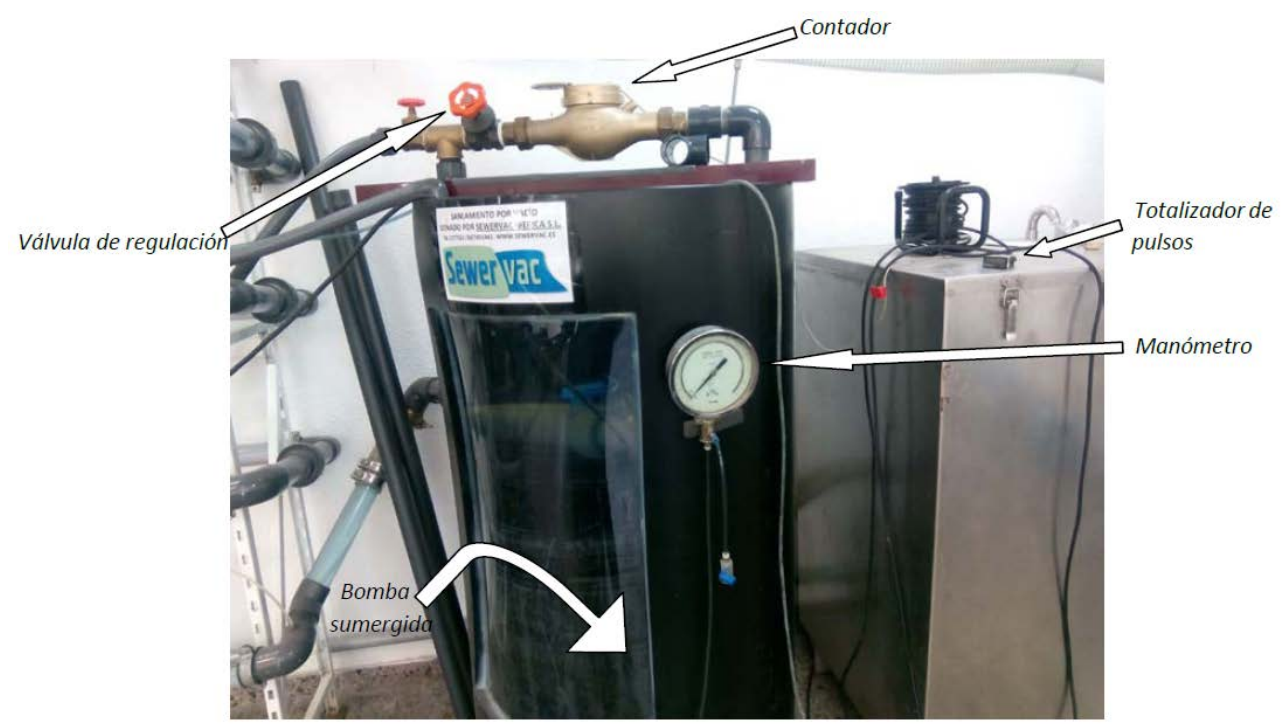

Fig. 3. Ejemplo de fotografía incluida en el apartado de descripción del banco de trabajo (Banco 2).

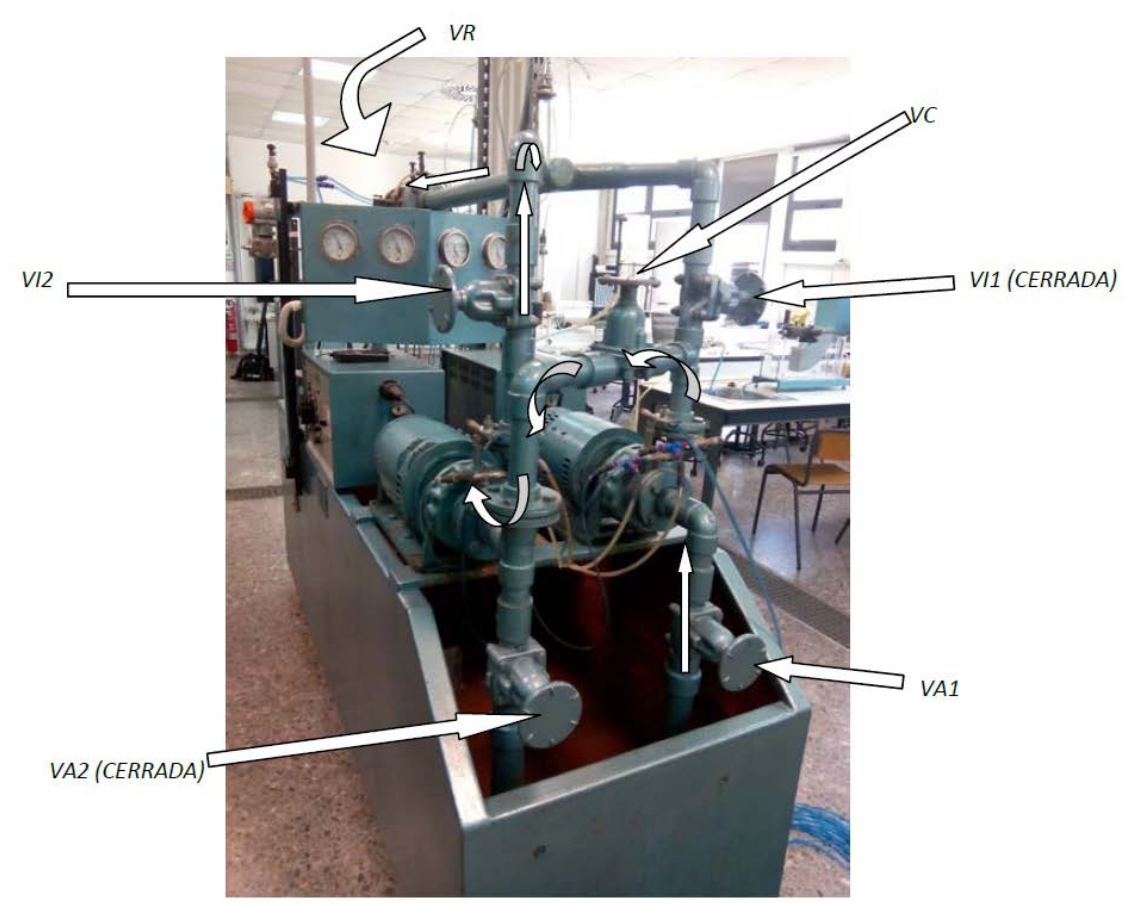

Fig.4. Ejemplo de fotografía incluida en el apartado de descripción del procedimiento a seguir (Banco 3, secuencia de apertura de válvulas en el ensayo de asociación en serie).

Ya se ha comentado previamente que debido a la diferencia que hay entre los distintos experimentos y los distintos bancos siempre ha sido un problema la aparición de "tiempos

(c)) 2016, Universitat Politècnica de València

Congreso In-Red (2016) 
muertos” durante el desarrollo de las prácticas. Esto se ha solucionado en parte con la necesidad de rellenar el anexo III con los resultados de los ensayos. Además y aunque no está incluido en el material de apoyo, cabe mencionar que las prácticas se completan, para cada uno de los tres grupos de una sesión (aprovechando los tiempos entre ensayos), con una demostración del fenómeno de cavitación y con una explicación de una exposición de diferentes elementos hidráulicos (ver Figura 5) que pueden ser observados y son comentados in situ con los alumnos.

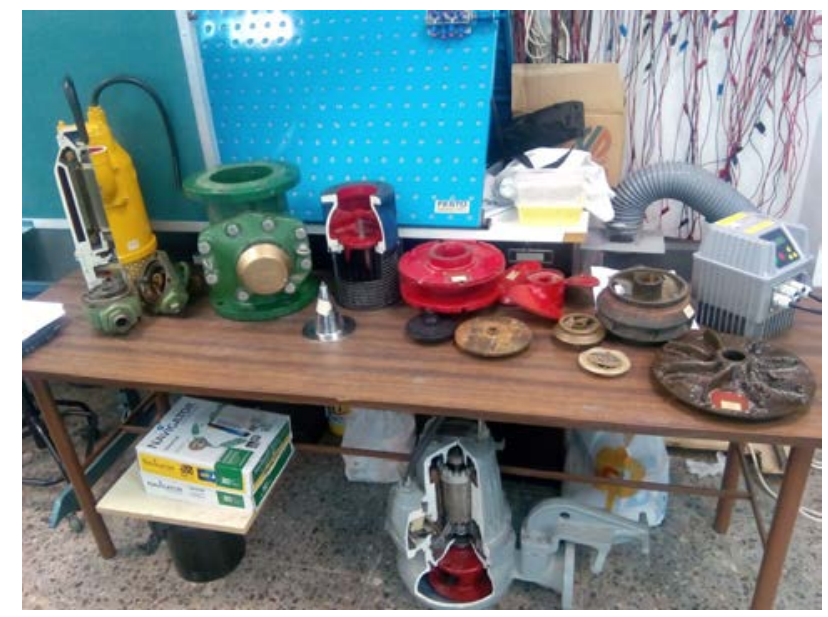

Fig.5. Exposición de elementos hidráulicos comentada durante las prácticas de laboratorio.

\section{Resultados}

La gran mayoría de alumnos (263 de 277, lo que supone casi el 95\% de los asistentes a las prácticas) entregaron los anexos de las partes I y II (Seguridad y Procedimiento respectivamente) al inicio de la clase, según lo requerido. No obstante, dado el elevado número de alumnos, evidentemente resulta complicado de evaluar el alcance del trabajo individual y la eficacia de este sistema de evaluación es limitada.

A decir verdad, la respuesta por parte de los alumnos ha sido algo menos activa de lo esperado, pero probablemente debido a la falta de concienciación del propio cambio de metodología. A pesar de las comunicaciones realizadas por los profesores y de las exigencias en las entregas, parece que los alumnos no acabaron de tomar conciencia del concepto de docencia inversa, por lo que, aunque efectivamente realizaron mayoritariamente las entregas de los anexos I y II, muchos de ellos reconocieron posteriormente, que no había preparado suficientemente las prácticas. De este modo, fue necesario explicar los fundamentos teóricos y los procedimientos de operación prácticamente con el mismo grado de detalle que en el caso de la docencia tradicional. A pesar de ello, el análisis comparativo de los resultados del curso anterior (2014, sin experiencia de docencia inversa) y del actual (2015, con docencia inversa) muestra una mejora en los resultados globales de la asignatura aunque apenas se detecte diferencia en la evaluación de las prácticas de laboratorio (ver Figura 6). 
En consecuencia, fue necesario ampliar el plazo de entrega de los tres anexos correspondientes a la parte III, puesto que en la mayoría de casos, no dio tiempo a cumplimentarlos durante la práctica, al consumirse un tiempo importante en las explicaciones. Los resultados en la evaluación de esta parte han resultado similares a las evaluaciones de resultados de años anteriores, si bien los del conjunto de la asignatura han mejorado, siendo uno de los principales objetivos de cualquier práctica, el ayudar a entender y asimilar los conceptos estudiados en la parte teórica de la asignatura.

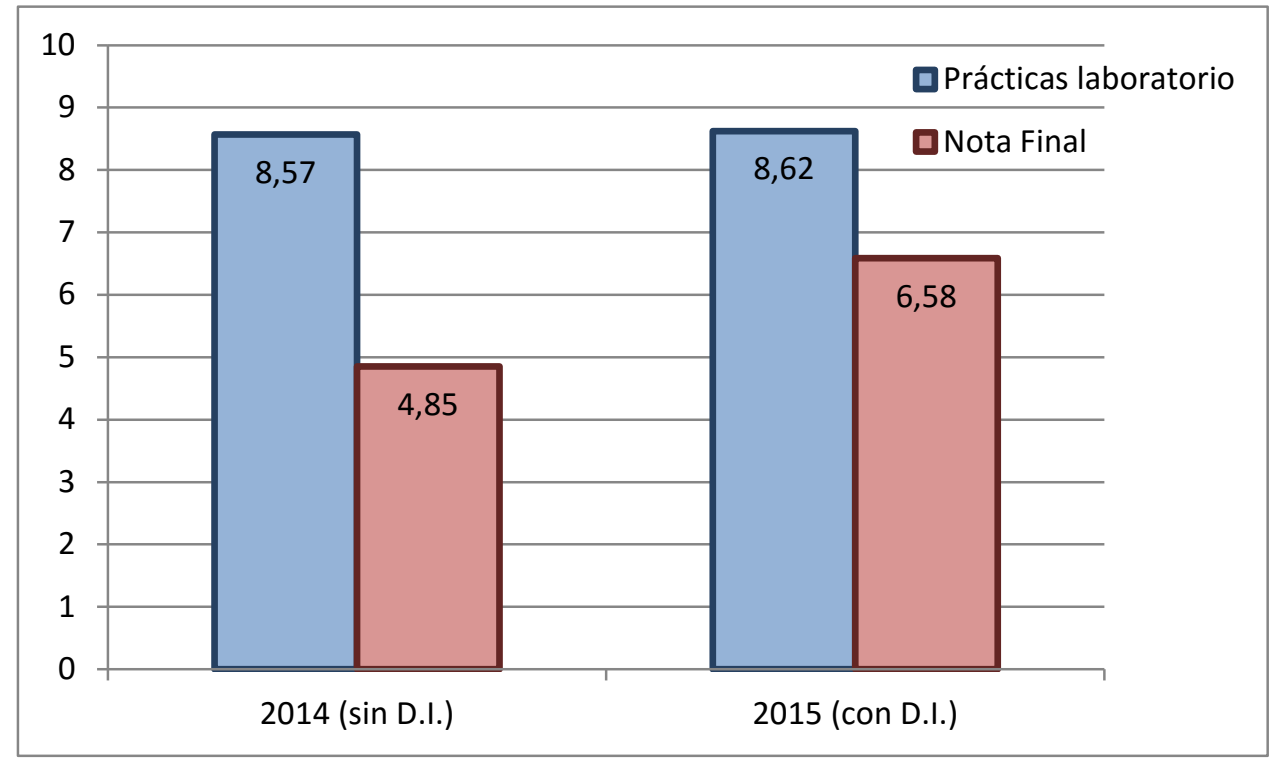

Fig.6. Comparación de los resultados académicos de los cursos 2014-15 (sin experiencia de docencia inversa) y 2015-16, con docencia inversa).

Salvo por las cuestiones mencionadas, en conversaciones informales con algunos alumnos se pudo observar que la organización de las prácticas y el material de apoyo parecían en general ser percibidos de forma positiva. En concreto, algunos alumnos, aunque reconocieron no haberlo analizado con profundidad previamente, manifestaron que con ayuda del material era sencillo llevar a cabo los ensayos durante la práctica. En cualquier caso, tratándose del primer año de implantación el resultado global, aunque no cuantificado podría considerarse como satisfactorio.

\section{Conclusiones}

La experiencia ha resultado muy interesante, confirmándose la utilidad de la aplicación de la metodología a la asignatura, especialmente en las prácticas, por lo que el próximo curso se ampliará la experiencia a las prácticas informáticas.

El material de apoyo elaborado se considera adecuado y algunos alumnos lo valoraron muy positivamente, aunque para mantener su efectividad debe actualizarse cada año con cualquier 
cambio o variación que pueda introducirse en los montajes o procedimientos, por pequeños que sean.

En general, teniendo en cuenta algunas limitaciones que dificultan el proceso (como el elevado número de alumnos), el planteamiento para las prácticas de laboratorio se considera también adecuado. Igualmente, se ha tomado nota de las lecciones aprendidas que serán asimiladas como posibles mejoras para el próximo curso. Entre ellas, destacaríamos las siguientes:

- Es necesario concienciar a los alumnos de la importancia de preparar los contenidos previamente. Para ello deberá hacerse un esfuerzo dicáctico y de comunicación tanto directa en clase, como indirecta a través del correo electrónico.

- Estudiar la posibilidad de incluir en la evaluación una pequeña encuesta para intentar obtenter mayor retroalimentación de la percepción y valoración del alumno.

- Aumentar la concienciación del alumnado, insistiendo desde el inicio de la asignatura y a lo largo del semestre en el concepto de la docencia inversa para que los alumnos tomen conciencia del cambio de metodología.

- Con este fin, estudiar la posibilidad de incluir en la evaluación al inicio de la sesión, de un breve test sobre el procedimiento (parte II) de modo que asegure una mayor preparación de esta parte o incluso la realización preguntas orales de forma aleatoria que formen también parte de la evaluación.

\section{Referencias}

ARGENTE, E.; ESPINOSA, A. y GARCÍA-FORNÉS, A. (2015). "Experiencia de la aplicación de la metodología Flipped-Teaching en la asignatura Concurrencia y Sistemas Distribuidos”. En Congreso In-Red 2015, Universitat Politècnica de València. Disponible en < http://ocs.editorial.upv.es/index.php/INRED/INRED2015/paper/view/1544>

FUNG, F.M. (2015). "Using first-person perspective filming techniques for a chemistry laboratory demonstration to facilitate a flipped pre-lab”. Journal of Chemical Education, 92(9), 1518-1521.

LÓPEZ PATIÑO, G. y MARTÍNEZ SOLANO, F.J. (2001). Máquinas Hidráulicas. Valencia: Servicio de Publicaciones de la UPV.

RHODE, J. (2015). Flipped Classroom Resources from UT Austin. http://www.jasonrhode.com/flipped-classroom-resources-from-ut-austin [Consulta: 30 de marzo de 2016]

SAMS, A. y BERGMANN, J. (2013). “Flip Your Students' Learning”. Educational Leadership, 70(6), 16-20. 